\title{
Macro Adjustment Based Task Scheduling in Hierarchical Grid Market
}

\author{
Peijie Huang ${ }^{1}$, Hong Peng ${ }^{1}$, and Xuezhen $\mathrm{Li}^{2}$ \\ ${ }^{1}$ College of Computer Science and Engineering, South China University of Technology, \\ Guangzhou 510640, P.R. China \\ ${ }^{2}$ Department of Computer and Information Engineering, Guangdong Technical College of \\ Water Resources and Electric Engineering, Guangzhou 510635, P.R. China \\ scuthpj@yahoo.com.cn
}

\begin{abstract}
Hierarchical organization is suitable for computational Grid. Although a number of Grid systems adopt this organization, few of them have dealt with the task scheduling for the hierarchical architecture. In this paper, we introduce a hierarchical Grid market model, which maintains the autonomy of the Grid end users, but incorporates macro adjustment of Grid information center into hierarchical Grid task scheduling. Simulation experiments show that the proposed method can improve the inquiry efficiency for resource consumers and get better load balancing of the whole hierarchical Grid market.
\end{abstract}

Keywords: Grid computing, task scheduling, macro adjustment, hierarchical market.

\section{Introduction}

How to effectively match the Grid tasks with the available Grid resources is a challenge due to the dynamic, heterogeneous and autonomous nature of the Grid. One common solution is to design a hierarchical Grid market. Although a number of Grid models and mechanisms employ hierarchical organization [1], [2], few of them have dealt with the task scheduling for the hierarchical architecture. Many existing Grid literatures of task scheduling have been proposed and achieved better performance in a single Grid market [3], [4]. However, given the hierarchical market model, how to effectively schedule the Grid tasks remains an important issue.

Our solution is novel in the sense that we introduce a hierarchical Grid market model which maintains the autonomy of the Grid end users, but incorporates macro adjustment of Grid information center (GIC) into hierarchical Grid task scheduling, and thus achieve a better scheduling in hierarchical Grid market.

\section{The Hierarchical Grid Market}

The Grid system is a hierarchical architecture with several different levels of Grid markets. An example of two-level hierarchical Grid market is shown in Fig. 1. 


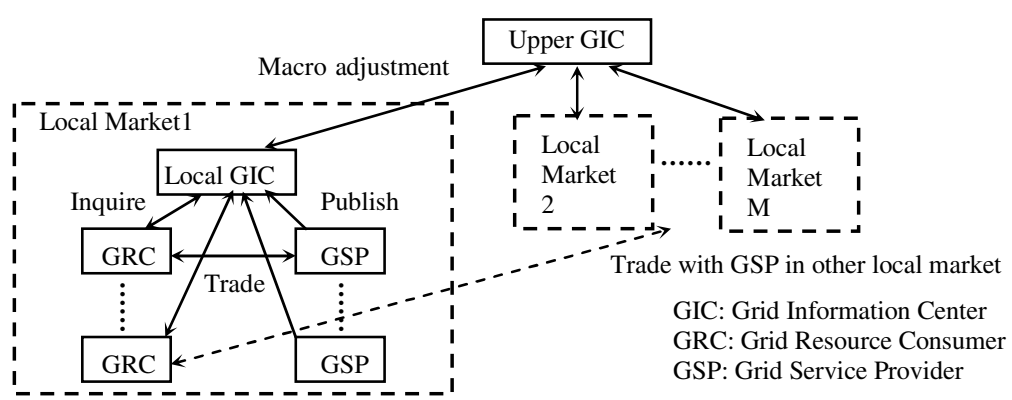

Fig. 1. The hierarchical Grid market

Each lowest level market covers certain local network area. In the local Grid markets, Grid service providers (GSPs) and Grid resource consumers (GRCs) trade with each other autonomously. Each local Grid market has a local GIC that allows GSPs to publish their services in order to attract GRCs to inquire for their tasks. Our market model extends the capability of the GICs, which also store and analyze the aggregate demands and supplies of their local Grid markets.

The network environment covering larger network area forms an upper Grid market, which consists of several lower-level Grid markets. Each upper Grid market has an upper GIC. The upper GIC stores the location of the lower-level GICs included and the aggregate demands and supplies of the lower-level Grid markets, and thus can provide inquiry across different lower-level markets within it and make macro adjustment to the lower-level GICs in this upper Grid market.

\section{Macro Adjustment Based Grid Task Scheduling}

Here, we present a method providing macro adjustment by off-line analysis.

Suppose that there are $n$ different kinds of Grid resources, we can denote the supply and demand of each local market as an n-vector. During new statistical period, suppose that there are $s$ GSPs in local market $m$, and $p$ tasks submitted within local market $m$ during peak demand period. We consider the following iterative procedure for GIC $m$ to obtain new estimates of demand and supply, $D_{m}$ and $S_{m}$ :

$$
\begin{array}{cc}
s_{m i}=\alpha \cdot T \cdot \sum_{j=1}^{s} r_{j i}+(1-\alpha) \cdot\left(s_{m i}\right)_{\text {old }} & i=1,2, \cdots, n \\
d_{m i}=\beta \cdot \sum_{j=1}^{p}\left(u_{j i} \cdot t_{j i}\right)+(1-\beta) \cdot\left(d_{m i}\right)_{\text {old }} & i=1,2, \cdots, n
\end{array}
$$

where $s_{m i}$ and $d_{m i}$ are respectively the component of the vectors $S_{m}$ and $D_{m} . r_{j i}$ is the published resource scale of resource $i$ of GSP $j$, and $T$ is the duration of the peak demand period. $u_{j i}$ is the demand of task $j$ for resource $i$, with occupy time $t_{j i} . \alpha$ and $\beta$ are the factors between 0 and 1 to control the impact of the past estimates. 
The excess supply of local market $m$ is $Z_{m}=S_{m}-D_{m}$. For a certain resource combination $c$, we denote the demand ratio of this combination of the whole Grid market as an n-vector $D R_{c}$. Then, we can compute the excess supply capability for resource combination $c$ of local market $m, C_{m}^{c}$ as follows:

$$
C_{m}^{c}=\max \left\{0, \min _{R_{i} \in c}\left(z_{m i} / d r_{c i}\right)\right\}
$$

where $z_{m i}$ and $d r_{c i}$ are respectively the component of the vectors $Z_{m}$ and $D R_{c} . C_{m}^{c}$ is 0 indicates that local market $m$ lacks excess supply of certain kinds of resources in combination $c$.

In the hierarchical Grid market, local GICs search within its local market with priority. When there is not any available resource for a certain submitted task with resource combination $c$, suppose that there are $k$ local markets have not been searched, the probability that local market $i$ is chosen to inquire is,

$$
\rho_{i}^{c}=C_{i}^{c} / \sum_{j=1}^{k} C_{j}^{c} \quad i=1,2, \cdots, k
$$

In next section, we compare the performance of our method, macro adjustment based task scheduling (MA-Based in short) with two contrastive methods:

Random Selection: In this method, without macro-adjusted guidance, when the local GICs cannot find an appropriate GSP in their own local markets, they choose another local market randomly with uniform probability to run a secondary inquiry.

Least Loaded: In the Least Loaded method, the local GICs choose the least loaded local market to run a secondary inquiry. Note, however, that in real environment the information is usually not up to date. We use a parameter $p$ so that once a task is submitted to a local market, the load of that local market is updated only with probability $p$ (for the results presented in this paper we use $p=0.5$ ).

\section{Simulation Experiments}

We have developed a simulated Grid environment based on GridSim [3]. The simulated Grid system consists of 20 local markets. Each local market has 10 GSPs and random amount of GRCs, uniformly distributed between 200 and 1000. There are total 4 kinds of Grid resources in this simulated Grid system.

The experimental evaluations include the inquiry efficiency and the load balancing of the whole Grid market. The inquiry efficiency is measured by the average amount of local markets in which Grid tasks that need secondary inquiry inquire. Suppose that there are $M$ local markets. The load balancing of the whole Grid market is measured by the balance degree during peak service period, which is denoted as:

$$
B D=1-\sqrt{\frac{1}{M} \sum_{i=l}^{M}\left(O_{i}-\bar{O}\right)^{2}} / \bar{O}
$$

where $O_{i}$ is the resource occupy rate of local market $i$ during the peak service period. 


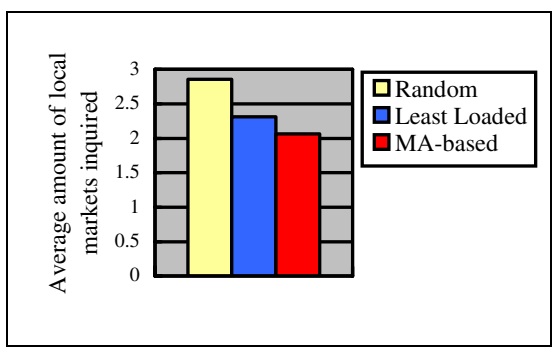

Fig. 2. The average amount of local markets in which Grid tasks that need secondary inquiry inquire

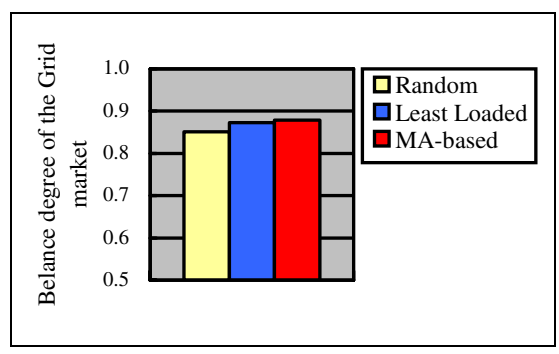

Fig. 3. The balance degree of the whole Grid market

First, we compare the inquiry efficiency. As we can see from Fig. 2, comparing to Least Loaded method, the performance gain of our method is about $15 \%$. This is mainly due to probabilistic failure to update the load levels after each task submission while using Least Loaded method. While comparing to Random Selection, the performance of our method gain reaches as high as $27.9 \%$.

We then observe the load balancing of the whole Grid market. As we can see from Fig. 3, our method guides most of the tasks to search in the local markets with big excess supply capability when they cannot be served in their own local markets, thus gets better load balancing than the contrastive ones. The Least Loaded method also outperforms the Random Selection. But it should pay for the large number of information exchanged between the upper GIC and the lower-level GICs.

\section{Conclusions}

This study is a first attempt to incorporate macro adjustment into Grid task scheduling. As we can see from the simulation results, our method outperforms the contrastive ones in improving the inquiry efficiency for resource consumers and getting better load balancing of the whole hierarchical Grid market.

Acknowledgements. This work is supported by the Provincial High-tech Program of Guangdong, China (A10202001, 2005B10101033).

\section{References}

1. Foster, I., Kesselman, C.: Globus: A Meta-computing Infrastructure Toolkit. International Journal of Supercomputer Applications, 1997

2. Chapin, S.J., Katramatos, D., Karpovich, J., et al: Resource Management in Legion. Future Generation Computer Systems, 1999, 15(5-6): 583-594

3. Buyya, R.: Economic-based Distributed Resource Management and Scheduling for Grid Computing. [Ph.D. Thesis]. Melbourne: Monash University, 2002

4. Li Chunlin, Li Layuan: QoS Based Resource Scheduling by Computational Economy in Computational Grid. Information Processing Letters. 2006, 98 (3): 119-126 